\title{
Parentesco, adscripción y crianza. Elaboraciones culturales de la adopción internacional y la circulación de niños ${ }^{1}$
}

\section{Kinship, Adscription and Child Rearing. Cultural Constructions of International Adoption and Circulation of Children}

\author{
Jorge GRAU REBOLLO \\ Grupo de Investigación en Antropología Fundamental y Orientada (GRAFO) \\ Departamento de Antropología Social y Cultural \\ Universitat Autònoma de Barcelona (UAB) \\ jordi.grau@uab.es
}

Recibido: 13 de septiembre de 2010

Aceptado: 29 de octubre de 2010

\section{Resumen}

La centralidad de los procesos de adscripción de niños y niñas ha ido dibujando cada vez con mayor nitidez la conveniencia de tomar en cuenta las formas diversas de circulación de niños como un fenómeno global de singular importancia en las estrategias de reposición del grupo y de cohesión social. Confinadas durante mucho tiempo a la etiqueta de "parentesco ficticio", algunas de las formas específicas de esta circulación, como la adopción internacional, fueron abriéndose paso en el panorama teórico a medida que los movimientos de menores bajo la legislación internacional se generalizaban y los debates sobre los corolarios económicos, éticos y políticos de los mismos ganaban terreno tanto en los foros académicos y profesionales como en los medios de comunicación. En este artículo reviso algunas de las líneas más relevantes en el panorama reciente del estudio de la circulación de menores en general y de las prácticas de adopción internacional como fenómenos de dimensiones globales, enfatizando la necesidad de prestar atención especial a las elaboraciones culturales de los procesos de adscripción y crianza en este ámbito.

Palabras clave: Adopción internacional, circulación de niños y niñas, antropología del parentesco, crianza, conceptos folk, etnografía, comparación transcultural.

${ }^{1}$ Este artículo recoge en parte los resultados de mi participación como investigador en el proyecto Teoría transcultural de la reproducción de los grupos humanos. La antropología del parentesco como estudio de los modelos socioculturales de procreación y crianza de los niños (SEJ2006-10864). Lo dirige la Dra. Aurora González Echevarría y lo financia el Ministerio de Educación y Ciencia (Plan Nacional de $\mathrm{I}+\mathrm{D}+\mathrm{i})$. 


\begin{abstract}
The relevance of children adscription has clearly suggested the need of taking into account the various forms of circulation of children considered as a global phenomenon of particular significance in the replacement strategies of a given group, as well as for its social cohesion. Confined for a long time to the label of "fictive kinship", some of the specific forms of this movement, such as international adoption, emerged in the theoretical arena as movements of children under international law blossomed and multi-faceted debates over their economic, ethical and political corollaries developed both in academic and professional forums and in mass media. In this article I address some of the most fruitful lines in the recent study of circulation of children and international adoption practices, both regarded as phenomena of global dimensions. I try to do this by emphasizing the need of paying special attention to cultural constructions of adscription processes and child rearing.
\end{abstract}

Keywords: International adoption, circulation of children, anthropology of kinship, child rearing, folk concepts, ethnography, transcultural comparison.

Referencia normalizada: Grau Rebollo, J. (2011). Parentesco, adscripción y crianza. Elaboraciones culturales de la adopción internacional y la circulación de niños. Revista de Antropología Social, 20, 31-54.

SUMARIO: 1. Introducción: la adopción internacional como constructo folk. 2. Aproximaciones académicas al estudio de la Adopción Internacional. 3. La regulación sociocultural de la procreación, la adscripción y la crianza de los niños y las niñas como dominio transcultural específico de la Antropología del Parentesco. 4. La relevancia transcultural de la circulación de menores. 5. Conclusiones. 6. Referencias bibliográficas.

\title{
1. Introducción: la adopción internacional como constructo folk
}

\begin{abstract}
Adoption is a prime example of conscious, volitional engineering in social life - it is a means for resolving conflict, on the one hand, and it is a process that may create conflict, on the other... Adoption offers a solution to a broad range of social, economic, and political problems that are likely to develop in small populations attached to island environments, including the insurance of equitable distributions of people relative to the requirements domestic and descent groups may have for heirs, balanced divisions of labor, sibling solidarity, enhanced political power, property alignments, and continuance of the groups themselves as corporate and cooperative entities (Brady, 1976: 288-289).
\end{abstract}

En los últimos veinte años, el interés social por la adopción internacional ha ido en aumento, lo que puede apreciarse tanto en la proliferación de estudios académicos como en la cobertura que le dispensan los medios de comunicación o la práctica adoptiva por personas solas y parejas que han iniciado procesos de adopción internacional.

El incremento en los procesos internacionales de adopción es fácilmente perceptible en una simple revisión estadística. Por ejemplo, según datos de Peter Selman (2006) para los años 1998 y 2004 las tasas brutas de adopción de países receptores, calculadas sobre la base del número total de adopciones constituidas por cada 
100.000 habitantes, permiten examinar la magnitud, el volumen total y la incidencia relativa de cada guarismo en el conjunto demográfico al que hace referencia — sean Estados o países con soberanía jurídica plena en la legislación del proceso adoptivo y su constitución definitiva, o comunidades con capacidad legislativa propia y reconocida pero dependientes en último término de estructuras políticas, jurídicas y administrativas superiores-. Por ejemplo, en 1998, Noruega, con una tasa bruta de adopción de 14.6, es seguida a una relativa distancia por Dinamarca - 11.8- y Suecia - 10.5- España ocuparía el décimo lugar en el ranking con una tasa del 3.8. Seis años después, Noruega se mantendría en primer lugar - 15.4-, seguida ya de cerca por España - 13.0 - y Suecia - 12.3- De los 15 países que conforman la tabla, hasta diez de ellos mostrarán un incremento significativo de dicha tasa respecto a su valor anterior. Si además tuviésemos en cuenta el volumen total de población en cada país, apreciaríamos mejor la proporción relativa que alcanzan las adopciones internacionales en cada uno de ellos ${ }^{2}$.

En algunos países, como España, que ingresaron con cierta demora en el pool de países de recepción, el incremento fue vertiginoso. Entre 1997 y 2003 se adoptaron en España, en números absolutos, 18.501 menores; pasando de un total de 942 adopciones internacionales en 1997 a 3951 en $2003^{3}$, lo que supone un incremento del $319,4 \%$ en 7 años. En un periodo similar, en los Estados Unidos, según fuentes de la Office of Children's Issues, United States Department of State (2010), el incremento entre 1999 y 2003 fue del 37,76\%, pasando de 15.719 adopciones en 1999 a 21.654 en 2003.

Con todo, la magnitud de adopciones registradas — aquellas que se formalizan a partir de procedimientos jurídicos sancionados tanto por las respectivas legislaciones nacionales de los países de origen y destino del menor como de las normativas y directrices internacionales pertinentes - no se aprecia únicamente en la contundencia de las cifras ${ }^{4}$, sino que impregna los debates sociales y abre nuevos escenarios teóricos acerca de la exclusión, la migración, el racismo o las nociones folk respecto al rol que juega la idea de sustancia en la confección de la identidad, poniendo en algunos casos de relieve la emergencia de nuevas formas de esencialismo vinculadas a viejos debates sobre el peso de la biología, la adscripción o la noción de ciudadanía (Howell, 2006; Howell y Melhuus, 2009). Paralelamente, se movilizan inquietudes acerca, por ejemplo, de la influencia que puedan ejercer ciertas decisiones e intereses económicos y políticos en la delineación de nuevas correlaciones de poder entre países ricos y potencias emergentes (Kim, 2007).

${ }^{2}$ Así, Noruega disponía en 1998 de 4.431 .464 habitantes (Official Statistics of Norway, 1999); Dinamarca contaba con 5.294.860 — según fuentes del Statbank—y Suecia se mantenía ligeramente por debajo de los nueve millones — 8.854.322, según cifras de Eurostat-.

${ }^{3}$ Esta información está disponible en: http://www.mtin.es/SGAS/FamiliaInfanc/infancia/Adopcion/Adopcion.pdf. Fuente: Ministerio de Trabajo y Asuntos Sociales.

${ }^{4}$ Las podemos encontrar desglosadas en función de diversos parámetros en Choulot et al (2005); Engel et al. (2007); Kane (1993); Mohanty y Newhill (2006); Selman (2006, 2007, 2009); y Weil (1984). 
En esta línea, los acogimientos y adopciones internacionales permiten, en su dimensión histórica, rastrear el pulso político de las relaciones internacionales, entre ellas el historial bélico de las grandes potencias coloniales del llamado primer mundo - tanto en las confrontaciones que mantuvieron entre sí, por ejemplo, los flujos adoptivos que siguieron al término de la Segunda Guerra Mundial, como en las contiendas que enfrentaron a cada una de ellas con otros países, frecuentemente en el marco de estrategias políticas dibujadas por estas potencias a escala global, como es el caso de Estados Unidos y Corea o Vietnam-. Algunos de los otrora países de origen fueron gradualmente convirtiéndose en países de destino para los menores adoptados, dibujando nuevas topografías del poder en los escenarios geopolíticos mundiales (Fonseca, 2002).

También es patente la relevancia que se concede al fenómeno adoptivo desde disciplinas tan variadas como la Psicología, la Demografía, la Antropología Social, la Pediatría, el Trabajo Social o el Derecho; no exclusivamente por afectar a centenares de miles de personas en todo el mundo - sólo restringiéndonos a las adopciones jurídicamente constituidas - e implicar a múltiples cuerpos legales, instituciones oficiales y agrupaciones de colectivos implicados, ni por el impacto que pueden suscitar en el escenario político internacional, sino fundamentalmente por la fecundidad heurística de su estudio y por las confluencias interdisciplinares a que da pie. En el seno de cada una de estas disciplinas se ramifican también las conexiones teóricas con ámbitos y dominios distintos, promoviendo y alentando las confluencias e intersecciones entre ellos. Por ejemplo, desde la Antropología Social y Cultural, Linda Seligmann, en su revisión de los volúmenes editados por Bowie (2005) y Volkman (2005), alude al: "uncharted territory and cultures that transnational adoption is fast creating" (2006: 545), a los que Volkman se había referido como las nuevas geografías del parentesco (2005: 2), vinculando el fenómeno de la adopción internacional a contextos políticos, económicos y sociales, así como a las políticas reproductivas estatales - donde las haya - cuestionando las fronteras ideológicas impuestas por conceptos como "cultura", "raza", "etnicidad", "nación" o clase. Otro tipo de fronteras, las biológicas, han sido también aproximadas desde la Antropología del Parentesco, singularmente en lo que refiere a las tecnologías de reproducción asistida (Marre y Bestard, 2004) y a las reflexiones relacionadas con su condición de paliativo de la insuficiencia biológica, mecanismo de completud social - establecimiento de una familia a partir de la progenie- Otras derivaciones, desde la antropología, conectan el parentesco con la movilidad social de los individuos, las prácticas políticas o los cuerpos jurídicos (Briggs y Marre, 2009).

A grandes rasgos, buena parte de investigaciones desde diversas disciplinas se centran en análisis sectoriales del proceso de adopción, tratando de dar cuenta en profundidad de cada etapa del itinerario adoptivo y de explorar meticulosamente cada una de sus ramificaciones. La combinación de estas investigaciones permite conocer el fenómeno con mayor profundidad, hacerlo inteligible, orientar su práctica presente y anticipar, en la medida de lo posible, problemáticas futuras.

En este artículo propongo encarar la cuestión más allá del ámbito restringido de los procesos jurídicos y de nuestras concepciones folk al respecto, que constituyen, 
en último término, el sustrato básico sobre el que concebimos y proyectamos el concepto "adopción", entendiendo las adopciones internacionales como formas específicas de circulación de menores, cuya morfología, dimensión, causas, estímulos y personas implicadas varían de una cultura a otra, pero donde pueden establecerse algunos rasgos comunes, entre los que destacan la mejora social de los individuos implicados - no sólo los menores-, su condición de estrategia para la relacionalidad social y, en conexión con ambas, su carácter táctico para la cohesión social.

Para ello, debemos ampliar nuestra perspectiva y entender estas adscripciones como hechos sociales que trascienden el estricto itinerario socio-jurídico adoptivo, para poner en conexión, en origen y destino, los espacios sociales en los cuales se lleva a cabo, los contextos y circunstancias en que se realiza, los agentes implicados, en diversa medida, a lo largo del proceso y a las consecuencias sociales que comporta. Esto supone encararlas como el resultado de elaboraciones culturales específicas, similares en algunos rasgos, profundamente distintas en otros; pero significativas por sí mismas en el entramado relacional de cualquier grupo humano.

Desde el GETP/GRAFO proponemos hacerlo desde una concepción de la Antropología del Parentesco que entiende como su dominio transcultural específico la regulación sociocultural de la procreación, la adscripción y la crianza de niños y niñas ${ }^{5}$, línea que he venido desarrollando en los últimos años en el ámbito de la circulación de menores.

La relevancia de esta circulación constituye la columna vertebral de este artículo. Sin embargo, antes de detenerme en consideraciones teóricas y fundamentos etnográficos al respecto, creo necesario revisar someramente algunas de las aproximaciones disciplinares más fecundas de los últimos años a propósito de la adopción internacional, entendida en un sentido conceptualmente más restringido, aunque su influencia teórica y peso en el panorama académico aún predomina claramente en las aproximaciones disciplinares a las prácticas diversas de emplazamiento infantil. A continuación enfocaré la regulación sociocultural de la procreación, la adscripción y la crianza de los niños y las niñas como dominio transcultural específico de la Antropología del Parentesco para, acto seguido, sostener la importancia de la circulación de menores como estrategia fundamental en muchas sociedades para la organización social. Defenderé también el interés de la comparación de estos fenómenos diversos de circulación — muchos de ellos considerados informales - para una mayor y más completa comprensión de las estrategias sociales de reproducción, así como para guiar la construcción de modelos etnográficos procreativos concretos.

Por último, subrayaré la centralidad de la etnografía para conectar cada una de las etapas vinculadas y confluyentes en los cambios sociales de adscripción de menores, obligando a restringir los supuestos teóricos e ideológicos que dan cuerpo a nuestras propias elaboraciones folk y prestando atención a las elaboraciones culturales más amplias que cada sociedad genera respecto a: (a) las ideas, normas y usos relativos al proceso procreativo; (b) la adscripción de los niños y las niñas;

${ }^{5}$ Véase: González Echevarría et al. (2010); González Echevarría, Grau y Vich (2010); y Grau (2004, 2006). 
(c) su cuidado hasta que alcanzan la madurez social; (d) las representaciones que vertebran los procesos de procreación, adscripción y crianza; y (e) las relaciones, sentimientos culturalmente pautados y representaciones que se generan e instituyen a partir de los procesos de procreación, adscripción y crianza entre las personas implicadas en ellos, y que se desarrollan a lo largo de la vida (González Echevarría et al., 2010: 102 y ss.).

\section{Aproximaciones académicas al estudio de la Adopción Internacional}

Revisaré a continuación, sin ánimo de ser exhaustivo, algunas de las líneas más fecundas de investigación con alusión a algunos de los trabajos más relevantes de las mismas. Lógicamente, cada disciplina ha trabajado y trabaja en líneas distintas y complementarias respecto a la adopción internacional y es imposible aquí sintetizarlas todas. Pero sí me gustaría mencionar algunas de las que se han mostrado más fructíferas en los últimos años ${ }^{6}$.

En congruencia con el motto fundamental que ampara el procedimiento jurídico de la adopción internacional, el bienestar del menor como fin último del proceso adoptivo ha guiado - $\mathrm{y}$, en buena medida, lo sigue haciendo - gran parte de la investigación desde diversos ámbitos académicos y profesionales. Así, por ejemplo, la adaptación —o maladaptación — social, a diversos niveles, de menores adoptados y los prejuicios sociales asociados al estigma de la adopción en determinados lugares o épocas históricas han marcado una línea fértil de investigación (Berástegui, 2005; Beverly et al., 2008; Bray, 1999; Dunn et al., 2000; Fernández Molina, 2002; Grotevant et al., 2000; Hanigan y Heitfield, 2001; Hübinette y Tigervall, 2009; Miall, 1987; Mohanty y Newhill, 2005; o Wegar, 2000).

Específicamente desde el ámbito psicológico, uno de los más prolíficos a este respecto, además de la exhaustiva revisión de la investigación disciplinar que han llevado a cabo Jesús Palacios y David Brodzinsky (2010), cubriendo desde mediados de los años cincuenta del siglo XX hasta la actualidad, a propósito de la población adoptada internacionalmente ${ }^{7}$, también se han abordado las consecuencias psíquicas para el menor, así como los procesos truncados de adopción (Abadi, 1989; Berástegui, 2003), y desde diversos ámbitos se ha aproximado la adopción — sus implicaciones - por parte de personas homosexuales, analizando sus constricciones jurídicas, su recurso como estrategia para conformar familias, las reivindicaciones que en ocasiones se invocan y los paralelismos ideológicos con otras formas de paternidad y maternidad (Abolafio y Rubio, 2004; Cadoret, 2003; López Sánchez,

${ }^{6}$ Tampoco puedo acometer aquí un recorrido histórico de la adopción. Hay estudios, tanto parciales como más comprensivos, que, además, proporcionan en algunos casos un compendio bibliográfico en cada disciplina delineando las líneas maestras de investigación al respecto (véase, por ejemplo: Bartholet, 2005; Fisher, 2003; Freundlich, 2002; Goody, 1969; Jociles y Charro, 2007; Palacios y Brodzinsky, 2010).

${ }^{7}$ En ella destacan tres grandes tendencias: la primera de ellas se dirigió a la comparación entre adoptados y no adoptados con el transfondo de la normalidad como referente; la segunda incidiría en la recuperación de los indicadores de normalidad, una vez se ha superado la adversidad inicial; la tercera y última referiría a los procesos y factores subyacentes al ajuste y desarrollo familiar en la adopción (Palacios y Brodzinsky, 2010). 
2006; Moreno y Villa, 2007; Ocón, 2002; Renaud, 2007; o Ricketts y Achtenberg, 1990).

A otros niveles, la salud física y mental de los menores adoptados ha sido también un foco destacado de interés. Así, encontramos, entre otros, estudios sobre presuntos diferenciales en la inversión económica de los padres en la monitorización y el tratamiento de salud entre hijos biológicos y adoptados (Case y Paxon, 2001; o Daly y Wilson, 1985); reflexiones acerca de los efectos perniciosos de la institucionalización en los retrasos detectados en menores adoptados (Johnson, 2002); análisis de la salud mental y adaptación en jóvenes y adolescentes procedentes de adopciones internacionales (Lindblad et al., 2008); mención a conductas suicidas en adoptados internacionales al llegar a la etapa adulta (Borczyskowski et al., 2010); predicciones sobre futuras conductas antisociales (Grotevant et al., 2006); o estudios de desarrollo físico y cognitivo tras procesos de adopción internacional (Dries et al., 2010); además de ensayos sobre el rol que desempeñan la genética y la intervención desde la biomedicina (Lebner, 2000; entre otros).

La trascendencia de la esfera legal es apreciable en el interés teórico en los cuerpos jurídicos sobre adopción y derechos de los adoptantes y adoptados (Adroher, 2007, 2008, 2009; Martínez de Aguirre, 2001; Polaino, Sobrino y Rodríguez, 2001) o la presumible colisión de prácticas tradicionales de emplazamiento a nivel local con el ordenamiento jurídico de la adopción internacional (Fonseca 2002, 2006; Roby y Matsumura, 2002).

También han sido objeto de estudio cuestiones relativas a la percepción y conformación identitaria entre los adoptados, especialmente en comparación con procesos análogos en hijos que son criados por sus progenitores biológicos, poniendo en juego el peso de los referentes étnicos y subrayando los problemas específicos que conciernen al establecimiento de puentes emocionales efectivos entre padres adoptivos y menores adoptados (Noy-Sharav, 2005). En este sentido, la atención se ha fijado, además, en cuestiones relativas a la identidad racial y étnica en las relaciones entre padres y madres adoptivos y menores adoptados, con especial énfasis en la adopción transracial; el interés se ha extendido, entre otras cosas, al papel que las narrativas e imágenes de los padres adoptivos construyen en su derredor y a las consecuencias que pueden comportar en la conformación de una identidad étnica en los menores (Briggs, 2003; Dorow, 2006; Grice, 2005; Silverman, 1993; Westhues y Cohen, 1998).

En esta misma línea, aunque a diferentes niveles, se ha informado acerca de la construcción de roles paternos y maternos y las prácticas socioeducativas (Amorós, 1986; Jociles y Charro, 2008) y se ha explorado la relación entre adopción, origen étnico y grupos de riesgo en la adopción internacional (Briggs y Ortiz, 2003). Ello conduce a reflexiones acerca de la conformación y vivencia de la identidad étnica, nacional y familiar en ámbitos distintos pero complementarios: reconceptualizaciones de sustancia, emparentamiento y transubstanciación (Howell, 2006) que, de algún modo, apuntan en ocasiones a los límites del esencialismo biológico o culturalista, y la relación intencional que se construye entre identidad - singularmente 
nacional y étnica-, circulación de flujos económicos e implicaciones políticas (Kim, 2007, Yngvesson, 2002).

Otras investigaciones han subrayado el rol del género a diversos niveles. Por ejemplo, David Eng (2006) ha acentuado la omisión de la figura de las niñas adoptadas en los debates sobre la situación de las mujeres en la nueva economía y en relación a formas distintas de explotación, como la prostitución femenina - fuente, recuerda Eng, de provisión de un número considerable de menores para los circuitos adoptivos_- enfatizando la esfera emocional. Kapp et al. (2001) se han fijado en el diferencial de posibilidades de recibir malos tratos en función del género del menor adoptado — siendo, afirman, ligeramente mayor en el caso de las niñas - . Kay Ann Johnson ha incidido en las consecuencias de no nacer varón en el seno de un Estado con una rígida política de planificación familiar, orientada a la unigenitura, en un contexto donde herencia y sucesión se rigen por un marcado sesgo patrilineal. Así, ha expuesto las condiciones de institucionalización y crianza de niñas en los orfanatos chinos (1993) y ha profundizado, junto con otros investigadores, en otros efectos de este diferencial de género en la consideración distinta de niños y niñas en China que conduciría, en última instancia, a la circulación invisible de estas últimas — missing girls — más allá de los canales públicos y oficiales (Johnson et al., 1998).

Por último, otras derivaciones académicas se han ocupado de las adopciones internacionales como formas específicas de fenómenos migratorios (Lovelock, 2000; Volkman, 2003; o Weil, 1984), o se han detenido en el análisis de procedimientos legales y prácticas ilegales en la abducción de menores - caso de las conocidas como stolen generations en Australia (Valentine y Gray, 2006) o los secuestros infantiles (Smolin, 2006) - , marco donde la percepción del país de origen se asocia, en la esfera de países receptores, con corrupción y tráfico incontrolado de bienes y personas (Hoelgaard, 1998), alimentando en ocasiones narrativas sobre tráfico de órganos y prostitución infantil (Campion-Vincent, 1997; Scheper-Hugues, 1996).

\section{La regulación sociocultural de la procreación, la adscripción y la crianza de los niños y las niñas como dominio transcultural específico de la Antropolo- gía del Parentesco}

Todas estas investigaciones inciden, en distinto grado y diverso alcance, en aspectos sectoriales del itinerario adoptivo, profundizando y promoviendo su imprescindible conocimiento y abriendo nuevos interrogantes teóricos que perfilan escenarios de análisis sugerentes y pertinentes en el ámbito de la adopción internacional. Mi propuesta aquí es enfocar el fenómeno desde una perspectiva transcultural, aprovechando el bagaje crítico de la Antropología del Parentesco, sobre todo desde los pasados años sesenta, y recurriendo a la producción etnográfica como fuente de provisión de datos y eje de reflexión. Así, desde el GETP/GRAFO, he trabajado en los últimos años en la extensión transcultural de la circulación de menores (Grau, 2006) - de la cual la adopción internacional constituiría una de sus formas específicas-, la posible inadecuación transcultural de un concepto - adopciónbasado en parámetros ideológicos y jurídicos folk (Grau, 2004), y en el fundamento etnográfico de la circulación de niños y niñas como un mecanismo para el provecho 
multilateral dentro de un grupo y la cohesión social en su interior $(2010,2011)^{8}$. Me ha interesado especialmente la comparación cultural, no la mera yuxtaposición de casos etnográficos, siguiendo la línea trazada en momentos y contextos distintos por Goody (1969) y Lallemand (1993). Para ello, he llevado a cabo una prospección etnográfica de archivo - eHRAF World Cultures - sobre la etnografía existente al respecto para 120 sociedades de todo el mundo y he revisado también bibliografía académica relevante de distintas fuentes disciplinares (Grau, 2010), lo que ha permitido integrar el análisis de la circulación de menores, en sentido amplio, en la revisión de las teorías del parentesco con el propósito de contribuir a la construcción de modelos procreativos generales en sociedades concretas. De este modo, la comparación entre formas de circulación de menores en grupos y etapas históricas distintas, puede orientar en el futuro investigaciones sobre modelos etnográficos más precisos.

Este análisis comparativo, crítico, procura, sin renunciar en absoluto al valor heurístico del concepto adopción, limitar los efectos perniciosos de una proyección etnocéntrica del mismo, basada en supuestos ideológicos y teóricos folk, sobre el espectro etnográfico. A partir de aquí, se trata de enfocar la adscripción de menores como un elemento caudal de la regulación sociocultural de la procreación que constituiría, como defendemos desde el GETP/GRAFO, el dominio central de la Antropología del Parentesco.

En esta propuesta, los niños y niñas aparecen no sólo como el capital básico de una sociedad de cara al futuro (Fox, 1967), sino como un ingrediente fundamental para la estrategia relacional presente y $\operatorname{cotidiana}^{10}$. En este sentido, hay dos antecedentes teóricos que me parecen relevantes. El primero de ellos es Claude Meillassoux (1977), quien refirió la movilidad de individuos adultos como base de la reproducción social. Esta movilidad respondería más a una necesidad cultural que biológica, en la medida que los individuos no solamente deben reproducirse sino también perpetuar la pertenencia a un grupo, lo que hace imprescindible el control de su descendencia. Para ello existen dos opciones: que las reproductoras circulen entre grupos a los que no pertenecen por filiación — ginecomovilidad - o que permanezcan en el grupo al que estén adscritas y exploten en él su potencial genésico — ginecoestatismo- . Es este supuesto básico de la circulación como engranaje social para la reproducción del grupo el que, entiendo, debería extenderse también a los niños y niñas, en el sentido que cada sociedad confiera a los menores susceptibles de ser englobados bajo el periodo de crianza. Es aquí donde entraría en juego el segundo precedente,

${ }^{8}$ Y no sólo en el interior del propio grupo, tal y como culturalmente se defina, sino también aprovechando contactos y relaciones diversas con grupos y colectivos foráneos, como el caso quinault, a propósito de un rumor surgido en 1909 en el sentido de que buscaban desesperadamente adoptar menores blancos para salvar sus tierras (Storm, 1990); los tukano, que incorporan grupos foráneos a sus fratrías mediante "adopción" (Arhem, 1981); o los nuer, de quienes Douglas Johnson asegura que usaron la adopción - junto al matrimonio - para garantizar su asentamiento al Este del Nilo, prefiriendo estas estrategias a la confrontación bélica con otros grupos (Johnson, 1994: 55).

${ }^{9} \mathrm{http}: / /$ ehrafworldcultures.yale.edu/ehrafe/

${ }^{10}$ Véase la investigación de Jessaca B. Leinaweaver (2008) sobre el recurso intencional a la circulación infantil en Perú, por ejemplo. 
que nos conduce a Suzane Lallemand (1993), quien sugirió cierta vinculación entre circulación infantil y alianza (Parkes, 2003: 744). Esta articulación nos permitiría redimensionar a los menores como un bien colectivo, desde la perspectiva de su propio grupo, que va más allá de la perpetuación de las líneas de filiación y descendencia o de la lógica de consolidación matrimonial. Su circulación se revelaría entonces como una parte esencial de la estrategia de individuos y grupos en lo que se refiere a relación, cohesión y mejora social.

Por esta razón, la crianza conduce nuestra mirada a los espacios sociales en los cuales se lleva a cabo, los contextos y circunstancias en que se realiza, los agentes implicados, en diversa medida, a lo largo del proceso y a las consecuencias sociales - en un plano más amplio que el del parentesco- que comporta. Desde esta óptica, en el GETP/GRAFO, hemos sostenido que el dominio de la Antropología del Parentesco:

... está constituido por los fenómenos socioculturales en tanto, y sólo mientras, los vemos desde la perspectiva de la reproducción del grupo por procreación y crianza de los niños, entendida como la reposición de los seres humanos que lo integran a través de la conceptualización y regulación de la generación, la adscripción de los niños, su cuidado, la atribución de la responsabilidad última de su socialización y enculturación hasta su madurez social y las relaciones que a partir de estos procesos se pueden desarrollar a lo largo de la vida (González Echevarría et al., 2010: 102).

De este modo, la Antropología del Parentesco se ocuparía, entre otras cosas, de las normas y usos relativos a la adscripción de los niños y niñas a grupos sociales y de los cambios de dicha adscripción - es decir: de su circulación-. El cuidado y responsabilidad sobre los niños nos obliga no sólo a atender a la perspectiva que define el dominio de la Antropología del Parentesco, sino que nos exige analizar sus confluencias y articulaciones con sistemas de género, acciones políticas y de control social sobre los individuos y nos invita a explorar las intersecciones con otros ámbitos de estudio de la Antropología cuando se estudian los mismos fenómenos desde otros ámbitos teóricos — por ejemplo el ejercicio de la sexualidad o los sistemas de creencias-.

\section{La relevancia transcultural de la circulación de menores}

La circulation des enfants m'est apparue comme un phénomène de grande ampleur: quasiment chaque unité domestique visitée pouvait citer l'exemple d'un don d'enfant au sein de la parenté. Des ces placements, certains apparaissaient transitoires, du type fosterage, alors que d'autres étaient définitifs, parfois officialisés (Laurence Pourchez, 2004: 30).

Un análisis detenido de la producción etnográfica revela la importancia que, no siempre explícitamente bajo la configuración de transferencias formales, ha tenido la movilidad infantil en las sociedades que han sido tradicionalmente objeto de atención por parte de la Antropología Social y Cultural. Incluso es rastreable esta centralidad en la historia reciente de Europa. En los últimos años, merced al vaciado 
exhaustivo de información etnográfica disponible en los eHRAF World Cultures y de la revisión de literatura académica y disciplinar al respecto (Grau, 2010), he podido apreciar la diversidad de formas de organización social bajo las cuales se transfiere, formal e informalmente, la adscripción de menores, así como apreciar el bien social e individual al que generalmente responde.

Como consecuencia de esta investigación, he procurado establecer una definición con un menor lastre folk que "adopción" y más susceptible de adecuarse a la pluralidad apreciable en la etnografía. Por ello, defino circulación de niños y niñas como la transferencia temporal o permanente, potencialmente reversible, de menores entre personas vinculadas o no previamente entre sí por lazos familiares y que pueden o no compartir responsabilidades sobre su cuidado y la autoridad sobre su comportamiento, teniendo en cuenta que esta transferencia, a menudo considerada "informal", suele comportar un cambio de residencia, puede tener efectos sobre la adscripción, herencia y sucesión en el seno de los grupos o familias receptores y no acostumbra a suponer ni la discontinuación de relaciones con los padres biológicos ni la incompatibilidad de filiación entre el núcleo de procedencia y el de emplazamiento.

Los propósitos sociales que persigue esta movilidad son diversos — desde corregir la infecundidad o infertilidad de individuos adultos hasta la conveniencia de incorporar varones para perpetuar el culto a la memoria paterna, pasando por la necesidad de aliviar cargas de sustento en la propia unidad familiar o la previsión de asegurarse cuidadores para el futuro, por ejemplo- y abarcan espacios y agentes igualmente distintos, si bien también presentan elementos comunes. Entre ellos, aunque no sólo, podemos destacar la mejora social de los individuos implicados —no sólo los menores - y la cohesión social que la acompaña (Grau, 2010) y su rol táctico para el estrechamiento de la relacionalidad social (Grau, 2011). Veamos dos ejemplos ilustrativos al respecto: el primero de ellos se refiere a los kanuri:

Whether or not Kanuri adoptions exist for the reasons given by informants, it is certain that they do create, maintain, and strengthen wider social relationships, giving them a solid basis for continuity through time. The links between two residence groups, either in the same kinship system or between different ones, are made concrete and real. The child, by living in intimate contact and having primary social relations both in his biological family and his adoptive family has widened his primary relationships and solidified a definite continuity into his own generation of one link or agent of his parents' relational network in the society (Cohen 1960: 156-157).

El segundo corresponde a los Igbo, quienes contemplan varias razones para la adopción como, por ejemplo, la mejora social de los hijos, el fallecimiento de la madre biológica o la esterilidad femenina (Forde, 1950). En este último caso, evitan dar alguno de sus hijos a parientes ancianos que no los tienen, ocupándose diversos miembros de la familia extensa de las necesidades de su granja (Meek, 1970). Sin embargo, esto no quiere decir que no exista la circulación de niños entre este grupo: Ottenberg (1980) sostiene que dicha circulación puede obedecer a tres motivos: (a) 
cuestiones de intendencia doméstica - llanto excesivo del bebé, cuidado inadecuado de su madre, etc.--; (b) "presentación" de un hijo: a partir de los seis años de edad, un menor puede ser "presentado" a otro hombre, familiar o conocido, con quien vivirá y a quién ayudará mientras conviva en su hogar, disfrutando de pleno estatus familiar en su nuevo emplazamiento, pero regresando a casa de su familia natal cuando lo desee; y (c) estrategia de mejora social: un menor puede ser ubicado con un hombre más influyente y poderoso, quien se beneficiará de su ayuda, pero al mismo tiempo le ofrecerá cobijo y le ayudará en su promoción social; como en casos anteriores, el menor puede decidir volver cuando quiera con su nueva familia ${ }^{11}$.

Cualquier aproximación a las elaboraciones culturales que acompañan la movilidad social de los menores debe confrontarse, en primer lugar, con nuestros propios términos y supuestos folk. Ya me he referido anteriormente a la fragilidad de adopción como concepto teórico cuando se aplica a la investigación transcultural, por cuanto es un término fuertemente lastrado por componentes jurídicos e ideológicos culturalmente específicos (Grau, 2004, 2006). Algunos de estos supuestos de base obligan a matices continuos en la traducción etnográfica. Por ejemplo, Lila AbuLughod (1993: 135), a propósito de los beduinos libios, sostiene que: "The women thought this amusing, though the idea of adoption-parents giving up their own children-horrified them". Precisamente a propósito de la extensión del fenómeno adoptivo, la propia Abu-Lughod comentaba unos años antes que los vínculos genealógicos no siempre resiguen la sangre y que esta forma de adopción se aplicaba tanto a individuos como a segmentos tribales enteros, lo que ocasionaba, prosigue, las quejas de los británicos. Todo ello prueba que la extensión del procedimiento adoptivo era notable en las primeras décadas del siglo XX (Abu-Lughod, 1986).

Entre los dogon, Bouju se refiere a "transferts d'enfants" e incluso en algún caso afirma que: "Nous ne savons pas ce que recouvre ici le terme adoptif" (1984: 84) y Huntingford, entre los Maasai, refiere que: "Under the term 'adoption' is also included the practice of adopting children within the family, e.g., a father will adopt a child of his wife's which has been begotten by another man, and a barren wife may adopt a child of one of her co-wives" (1953: 115).

Todavía en África, Turnbull sostiene que entre los mbuti: "Adoption is often agreed upon in order that a child, or even an adult, may return to his natal band, regardless of what kin remain to him there" (Turnbull, 1965: 131); y Helander señala su considerable extensión entre los somalíes:

Nevertheless, the extent to which adoption is practiced in southern Somalia is probably unparalleled among other peoples that claim to be formally constituted as groups by common ancestry. In the southern Somali case, adopted members of some clans actually outnumber the original founding nucleus of authentic descendants.

${ }^{11}$ No debe confundirse esta ayuda que presta el menor, con el estatus de sirviente: "The differentiating factors between the child who is "presented... and the "servant" child... seem to be that while in the former case closeness of kinship and a desire to help a relative are the predominating motives, in the latter the hope that a child will profit from the association is paramount. When a child is sent away because of problems of adjustment, he is commonly sent to a brother or sister rather than a more distant relative" (Ottenberg, 1980: 203). 
Yet the importance given to descent as a constituting principle is not in the least upset. Adopted members continue to claim genealogical links with their clans of ancestry, yet are considered to be members of an entirely different clan (Helander, 1988: 189).

Esta extensión puede cruzarse en algún caso, como el de los mismos somalíes, con los matices folk a la proyección etnocéntrica de nuestros supuestos culturales. Así, algunos miembros de clanes que han adoptado foráneos consideran problemática esta heterogeneidad frente a otros clanes somalíes. En algunos casos, parece incluso que el número de foráneos adoptados puede sobrepasar al de miembros nativos del clan, aunque:

A distinction is made between those born into the clan and those who have become members by adoption. These two categories are called "dhalad" and "dhaqan", "by birth" and "by culture"... The other category of adopted members are called "dhareer". They are joined through marriage links, and they are definitely the majority of all adopted members (Helander, 1988: 133; lo entrecomillado está subrayado en el original) ${ }^{12}$.

Igualmente, en Asia, por ejemplo, encontramos en las fuentes etnográficas alusiones a sociedades que la practican con frecuencia y también referencias a su escasa incidencia o carácter esporádico, aunque no siempre hay consenso al respecto para una misma cultura. Así, entre los thai centrales, Kamol Janlekha sostiene que: "The authenticity of the term 'adopted child' as used in the village [Bangchan] is nevertheless rather questionable. There has never been such a practice like legal registration of adopted children in the village, although this is provided by law" (1956: 64). Por su lado, Amyot (1920) afirma que ello es infrecuente. Y Jane Richardson Hanks sostiene que: "Adoption was fairly common, though not always of lasting satisfaction" (1963: 16). Entre los garo, Alan Playfair sostiene que: "With the exception of the adoption of a nokrom in the absence of a sister's son, there is no adoption among the Garos" (1909: 73), mientras que autores como Robbins Burling o Kumie Marak - quien se refiere más explícitamente a su importancia en el parentesco garo (1997: 104) - parecen sugerir lo contrario: "However, even if a couple should have no daughters, they can adopt a close relative who will come into their home and assume the duties of an actual daughter... An adoption is frequently arranged when the girl is as young as ten or twelve, and at a time when the girl can hardly have much choice in the matter" (Burling, 1963: 77, 78).

Y es precisamente esta facilidad y generalidad de la circulación de menores entre parientes y entre hogares la que en algunos grupos, como los thai centrales, condiciona la fidelidad de los censos en el aspecto genalógico. Kamol Janlekha (1956) ya señalaba que muchos sobrinos y sobrinas podían ser en realidad hijos/as "adoptados/as", pero oficialmente no se contaban como tales ya que sólo computaban como

${ }^{12}$ Helander matiza que dhareer significa "cartilago" — entre huesos—, un detalle significativo en este caso, ya que "hueso" es el nombre que reciben las agrupaciones de agnados, sugiriendo que el matrimonio es la forma idónea de vincular dos patrilinajes. 
adoptados aquellos a quienes los cabezas de familia mencionaban explícitamente como tales.

Estos matices pueden aplicarse también a sociedades de América del Norte, como los blackfoot, entre quienes, además de la frecuencia con que se llevan a cabo los emplazamientos, Lucien Hanks afirma que:

The practice of "bringing up" children of relatives adds further potential members to the original family. This practice is distinct from adoptions where the foster parents conceal the fact of adoption from the child. In "bringing up" a child, a middle-aged man and his wife merely take a grandchild to live with them... Both "bringing up" a child and adoption occur with considerable frequency (Hanks, 1950: 153).

Incluso se ha afirmado en algunos casos, como entre los tarahumara mexicanos, que en algunos lugares, casi la mitad de los menores no viven con sus padres biológicos (Passin, 1943), pudiendo darse no sólo adopciones sino incluso readopciones, por una tercera familia, de niños ya adoptados (Champion, 1963).

Una vez sometida a control la extensión de presupuestos específicos, debe tenerse en cuenta la dimensión de que los propios presupuestos culturales del grupo hayan quedado reflejados. Esto es algo que a menudo se acompaña del término nativo, que se traduce como adopción o adoptado, y de alguna precisión relevante en referencia a la forma en que se concibe la práctica a nivel local. Por ejemplo, entre los gusii africanos y en contraste con nuestra acepción de adopción:

\footnotetext{
... a child who has an ascertainable place in the lineage framework, but who is being brought up by people not his legal relatives - for instance at his mother's lover's home- is not an omogore or "bought person", but a "swallowed thing" (ekemerwa) or a "brought-in thing" (ekerentane). His status differs radically from that of the adopted child proper, in that he shares in none of the patrimonial rights of the foster-family. He is the person of whom elders foretell that he will "run home to father" when he wants to marry. To refer openly to the status of a "brought-in" child is considered insulting (Mayer, 1949: 25).
}

Por último, puede procederse a la comparación transcultural de rasgos homogéneos en la circulación de menores, siempre sobre la base que ofrecen $-\mathrm{y}$ las constricciones que imponen - las fuentes que manejamos a tal efecto.

Creo por ello que, en un nivel próximo a la adecuación de la práctica con nuestra perspectiva teórica en la definición de adopción como fenómeno específico, deben entenderse las adopciones como hechos sociales que trascienden el estricto itinerario socio-jurídico adoptivo, para poner en conexión, en origen y destino, los espacios sociales en los cuales se llevan a cabo, los contextos y circunstancias en que se realizan, los agentes implicados, en diversa medida, a lo largo del proceso, las elaboraciones que generan y las consecuencias sociales —en un plano más amplio que el del parentesco- que comportan.

A un nivel más general, si nos interesa una perspectiva más transcultural, debemos pensar en la circulación de menores como un conjunto de normas, prácticas y representaciones, con similitudes y diferencias entre culturas distintas, donde la 
responsabilidad última en el cuidado y la crianza de niños y niñas queda siempre bien establecida — con independencia del nivel de formalización en la movilidady cuyo emplazamiento y adscripción sirve tanto a intereses individuales como multilaterales en el seno de un grupo social - e, incluso, ocasionalmente, entre grupos distintos-.

\section{Conclusiones}

Buena parte de los estudios que sobre las prácticas adoptivas, específicamente en adopción internacional, se han llevado a cabo en las últimas décadas se dirigen principalmente a la comprensión profunda de las diversas fases que conforman el proyecto adoptivo, entendido como itinerario específico que, desde el plano jurídico, conforma un patrón determinado con variantes tipificadas. Algunos estudios apuntan también a las consecuencias y derivadas políticas, éticas y económicas de los fenómenos que envuelven a la adopción internacional.

Desde mi propuesta teórica en el seno del GETP/GRAFO, he tratado, por un lado, de limitar los efectos de la proyección etnocéntrica de supuestos, teóricos e ideológicos folk. Por otro, propongo trascender el análisis pormenorizado de las distintas fases de un itinerario adoptivo para subrayar la pertinencia de:

(a) la crianza nos obliga a prestar atención a los espacios sociales, con frecuencia cambiantes, en los cuales se lleva a cabo, los contextos y circunstancias en que se realiza, los agentes implicados, en diversa medida, a lo largo del proceso y a las consecuencias sociales —en un plano más amplio que el del parentesco- que comporta;

(b) la circulación de menores, en la forma en que la he definido aquí, enfatizando a los menores como un bien colectivo que va más allá de la perpetuación de las líneas de filiación y descendencia o de la lógica de consolidación matrimonial. Su circulación se revelaría entonces como una parte esencial de la estrategia de individuos y grupos en lo que refiere a relación, cohesión y mejora social;

(c) la conexión disciplinar de los itinerarios adoptivos, como formas específicas de adscripción de niños y niñas, con las ideas, normas y usos relativos al proceso procreativo, el cuidado de los niños hasta que alcanzan la madurez social, las representaciones que vertebran estos procesos y las relaciones que generan, de cada uno de los modelos etnográficos de parentesco, así como con características socio-demográficas del grupo o país de origen que afectan a los niños, y en el ámbito específico de las adopciones internacionales como ahora las entendemos la conexión de todos estos factores con los procesos que llevan a una declaración de adoptabilidad y de las representaciones de la adopción internacional en el país emisor, que pueden estar vinculadas a las formas tradicionales de circulación.

La extensión y variedad de elaboraciones culturales en torno al emplazamiento y a la adscripción infantil, formales e informales, en el espectro etnográfico, permiten subrayar en última instancia no sólo la centralidad de los menores como bien estratégico futuro para una sociedad, como ya subrayara Robin Fox en los pasados años sesenta, sino como un activo presente de primer orden. 


\section{Referencias bibliográficas}

ABADI, Dora

1989 Adopción. Del abandono al encuentro. Buenos Aires: Ediciones Kargieman.

ABOLAFIO, Estrella; RUBIO, Ma Jesús

2004 “Adopción y parejas de hecho". Portularia, 4: 231-240.

ABU-LUGHOD, Lila

1986 Veiled sentiments: honor and poetry in a Bedouin society. Berkeley: University of California Press.

1993 Writing women's worlds: Bedouin stories. Berkeley: University of California Press.

ADROHER BIOSCA, Salomé

2007 "The rights of the adopted child and the public family policies in intercountry adoption" en A. Allen et al. (eds.), The UN Children's Rights convention: Theory meets practice. Bruselas: Intersentia, 441-63.

2008 "Intercultural families. A challenge for european private international law. A perspective from Spain". INTAMS Review. Journal for the Study of marriage \& spirituality, 14: 220-30.

2009 "La protección de los mayores vulnerables: una cuestión pendiente", en E. Llamas Pombo (coord.), Nuevos conflictos del Derecho de familia. Madrid: La Ley, 719-757.

AMORÓS, Pere

1986 La adopción desde una perspectiva socioeducativa. Tesis doctoral. Facultat de Filosofia. Universitat de Barcelona.

AMYOT, Jacques

1920 Village Ayutthaya: social and economic conditions of a rural population in central Thailand. Bangkok: Chulalongkorn University Social Science Research Institute.

ARHEM, Kaj

1981 Makuna social organization: a study in descent, alliance, and the formation of corporate groups in the north-western Amazon. Uppsala, Stockholm: Academiae Upsaliensis.

BARTHOLET Elizabeth

2005 "International Adoption", en L. Askeland (ed.), Children and Youth in Adoption, Orphanages, and Foster Care. Westport: Greenwood Press, 107-131.

\section{BERÁSTEGUI, Ana}

2003 Las adopciones internacionales truncadas y en riesgo en la Comunidad de Madrid. Madrid: Consejo Económico y Social. Comunidad de Madrid.

BORCZYSKOWSKI, A. von; et al.

2006 "Suicidal behaviour in national and international adult adoptees A Swedish cohort study". Social Psychiatry and Psychiatric Epidemiology, 41, 2: 95-102. 
BOUJU, Jacky

1984 Graine de l'homme, enfant du mil. Paris, Nanterre: Societe d'ethnographie: Service de publication du laboratoire d'ethnologie et sociologie comparative, Universite de Paris X.

BRADY, Ivan

1976 "Adaptative Engineering: An Overview of Adoption in Oceania”, en I. Brady (ed.), Transactions in kinship: adoption and fosterage in Oceania. ASAO. Monograph, $\mathrm{n}^{\circ}$ 4. Honolulu: University of Hawai Press, 270-293.

BRIGGS, Laura

2003 "Mother, Child, Race, Nation: The Visual Iconography of Rescue and the Politics of Transnational and Transracial Adoption”. Gender \& History, 15, 2: 179-200.

BRIGGS, Laura; MARRE, Diana (Eds.)

2009 International Adoption: Global Inequalities and the Circulation of Children. New York, London: New York University Press.

BRIGGS, Laura; ORTIZ, Ana Teresa

2003 "The Culture of Poverty, Crack Babies, and Welfare Cheats The Making of the 'Healthy White Baby Crisis'. Social Text, 21, 3: 39-57.

BURLING, Robbins

1963 Rengsanggri: family and kinship in a Garo village. Philadelphia: University of Pennsylvania Press.

CADORET, Anne

2003 Padres como los demás. Homosexualidad y Parentesco. Barcelona: Gedisa.

CASE, Anne; PAXSON, Christina

2001 "Mothers and others: who invests in children's health?". Journal of Health Economics, 20: 301-328.

CHAMPION, Jean René

1963 A study in culture persistence: the Tarahumaras of northwestern Mexico. Ann Arbor: University Microfilms.

CHOULOT,J. J. ; et al.

2005 "International adoption in France". Archives de pédiatrie:organe officiel de la. Société française de pédiatrie, 12,6: 718-720.

COHEN, Ronald

1960 The structure of Kanuri society. Ann Arbor: University Microfilms.

DALY, M.; WILSON, M.

1985 "Child abuse and other risks of not living with both parents". Ethology and Sociobiology, 6: 197-210. 
DRIES, L.; et al.

2010 "Infants' physical and cognitive development after international adoption from foster care or institutions in China". Journal of Developmental and Behavioral Pediatrics, 31 2: 144-150.

EHRAF WORLD CULTURES, base de datos electrónica on-line. http://ehrafworldcultures. yale.edu/.

ENG, David

2006 "Political Economics of Passion: Transnational Adoption and Global Woman: Roundtable on Global Woman". Studies in Gender and Sexuality, 7, 1: 49-59.

ENGEL, Madeline; PHILLIPS, Norma K.; DELLACAVA, Frances A.

2007 "International Adoption: A Sociological Account of the US Experience". International Journal of Sociology and Social Policy, 27, 5-6: 257-270.

EUROSTAT: http://epp.eurostat.ec.europa/eu.

FISHER, Allen P.

2003 “Still 'Not Quite as Good as Having Your Own'? Toward a Sociology of Adoption”. Annual Review of Sociology, 29: 335-361.

FONSECA, Claudia

2002 "Inequality Near and Far: Adoption as Seen from the Brazilian Favelas". Law \& Society Review, 36, 2: 307-431.

FORDE, Darryl

1950 "Ibo (Igbo)", en D. I. Forde y G. I. Jones (eds.), The Ibo and Ibibio-speaking peoples of south-eastern Nigeria. London, New York: Published for the International African Institute by Oxford University Press, 9-65.

FOX, R.

1967 Kinship and Marriage. An Anthropological perspective. Harmondsworth: Penguin.

FREUNDLICH, Madelina

2002 "Adoption Research: An Assessment of Empirical Contributions to the Advancement of Adoption Practice". Journal of Social Distress and the Homeless, 11, 2: 143-166.

GONZÁLEZ ECHEVARRÍA, Aurora; et al.

2010 "Sobre la definición de los dominios transculturales. La Antropología del Parentesco como teoría sociocultural de la procreación”. Alteridades, 20, 39: 93-106.

GONZÁLEZ ECHEVARRÍA, Aurora; GRAU REBOLLO, Jorge; VICH BERTRAN, Júlia

2010 "Las adopciones internacionales como 'hecho social total". Periferia, 12: 1-12. Edición electrónica: http://www.raco.cat/index.php/ Periferia/article/viewFile/199661/267045.

GOODY, Jack

1969 "Adoption in Cross-Cultural perspective". Comparative Studies in Society and History, 11: 55-78. 
GRAU REBOLLO, Jorge

2004 "Parentesco y adopción. Adoptio imitatur naturam.¿Nature vs. Nurture?". Quaderns-e, ICA. 3/2004a.

2006 Procreación, género e identidad. Debates actuales sobre el parentesco y la familia en clave transcultural. Barcelona: Ediciones Bellaterra.

2010 "La circulación de menores desde una perspectiva transcultural", en V. Fons, A. Piella y M. Valdés (eds.), Procreación, crianza y género. Aproximaciones antropológicas a la parentalidad. Barcelona: PPU, 213-234.

En prensa

"Estrategias socioculturales de procreación y crianza: de la adopción internacional a la circulación de menores", en J. Grau, D. Rodríguez y H. Valenzuela (eds.), ParentescoS. Modelos culturales de reproducción. Barcelona: PPU.

GRICE, Helena

2005 "Transracial Adoption Narratives: Prospects and Perspectives". Meridians; Feminism, Race, Transnationalism, 5, 2:124-148.

GROTEVANT, H. D.; et al.

2006 "Antisocial Behavior of Adoptees and Nonadoptees: Prediction from Early History and Adolescent Relationships". Journal of Research on Adolescence, 16: 105-131.

HANKS, Jane Richardson

1963 Maternity and its ritual in Bang Chan. Ithaca: Cornell University, Department of Asian Studies, Southeast Asia Program.

HANKS, Lucien Mason

1950 Tribe under trust: a study of the Blackfoot Reserve of Alberta. Toronto: University of Toronto Press.

HELANDER, Bernhard

1988 The slaughtered camel: coping with fictitious descent among the Hubeer of southern Somalia. Uppsala: University of Uppsala, Department of Anthropology.

HOELGAARD, Suzanne

1998 "Cultural Determinants of Adoption Policy: A Colombian Case Study". International Journal of Law, Policy and the Family, 12: 202-241.

HOWELL, Signe

2006 The Kinning of Foreigners. Transnational Adoption in a Global Perspective. New York, Oxford: Berghahn Books.

HOWELL, Signe; MELHUUS, Marit

2009 [2007] "Race, Biology and Culture in contemporary Norway: Identity and Belonging in Adoption, Donor Gametes and Immigration", en P. Wade (ed.), Race, Etnicity and Nation. Perspectives from kinship and genetic. Oxford, New York: Berghahn, 53-72.

HUNTINGFORD, George Wynn Brereton

1953 The southern Nilo-Hamites. London: International African Institute. 
JANLEKHA, Kamol Odd

1956 A study of the economy of a rice growing village in central Thailand. Ann Arbor: University Microfilms.

JOCILES RUBIO, $\mathrm{M}^{\mathrm{a}}$ Isabel; CHARRO LOBATO, C.

2008 "Construcción de los roles paternos en los procesos de adopción internacional: el papel de las instituciones intermediarias". Política y Sociedad, 45, 2: 105-130.

JOHNSON Dana

2002 “Adoption and the Effect on Children's Development". Early Human Developement, 68: 39-44.

JOHNSON, Douglas Hamilton

1994 Nuer prophets: a history of prophecy from the Upper Nile in the nineteenth and twentieth centuries. Oxford, New York: Clarendon Press, Oxford University Press.

JOHNSON, Kay Ann

1993 "Chinese Orphanages: Saving China's Abandoned Girls". The Australian Journal of Chinese Affairs, 30: 61-87.

JOHNSON, Kay Ann; BANGHAN, Huang; WANG, Liyao

1998 "Infant Abandonment and Adoption in China". Population and Development Review, 24, 3: 469-510.

KANE, S.

1993 "The movement of children for international adoption: an epidemiological perspective”. Social Science Journal, 30, 4: 323-339.

KAPP, S. A.; MCDONALD, Th.; DIAMOND, K. L.

2001 "The path to adoption for children of color". Child Abuse and Neglect, 25: 215-229.

KIM, Eleana

2007 "Our Adoptee, Our Alien: Transnational Adoptees as Specters of Foreignness and Family in South Korea”. Anthropological Quarterly, 80, 2: 497-531.

LALLEMAND, Suzane

1993 La circulation des enfants en societe traditionelle. Prêt, don, échange. Paris : L'Harmattan.

LEBNER, Ashley

2000 "Genetic 'Mysteries' and International Adoption: The Cultural Impact of Biomedical Technologies on the Adoptive Family Experience". Family Relations, 49, 4: 371-377.

LEINAWEAVER, Jessaca B.

2008 The Circulation of Children: Kinship, Adoption and Morality in Andean Peru. Durham, New York: Duke University Press. 
LINDBLAD, Frank; et al.

2008 "Adopción internacional en Suecia: salud mental y adaptación social en adolescentes y jóvenes”. Infancia y Aprendizaje, 31, 2: 211-231.

LÓPEZ SÁNCHEZ, Félix

2006 "La adopción por parte de personas homosexuales", Informació Psicològica, 87: 8-20.

LOVELOCK, Kirsten

2000 "Intercountry adoption as a migratory practice: A comparative analysis of intercountry adoption and immigration policy and practice in the United States, Canada and New Zealand in the post W.W. II period". The International Migration Review, 34, 3: 907-949.

MARAK, Kumie

1997 Traditions and modernity in matrilineal tribal society. New Delhi: Inter-India Publications.

MARRE, Diana; BESTARD, Joan

2004 La adopción y el acogimiento. Presente y Perspectivas. Barcelona, Universitat de Barcelona: Publicacions i Edicions de la Universitat de Barcelona, Estudis d'antropologia social i cultural, 13.

MARTÍNEZ DE AGUIRRE, Carlos

2001 "La adopción, entre los derechos del adoptado y los deseos de los adoptantes", en A. Polaina, A. Sobrino Morrás y A. Rodríguez Sedano (eds.), Adopción. Aspectos psicopedagógicos y marco jurídico. Barcelona: Ariel, 177-193.

MAYER, Ph.

1949 The lineage principle in Gusii society. London, New York: Oxford University Press.

MEILLASSOUX, Claude

1977 [1975] Mujeres, graneros y capitales. Madrid: S.XXI.

MIALL, Charlene

1987 "The Stigma of Adoptive Parent Status: Perceptions of Community Attitudes toward Adoption and the Experience of Informal Social Sanctioning". Family Relations, 36, 1: 34-39.

MINISTERIO DE TRABAJO Y ASUNTOS SOCIALES

s. a. Adopción en España. http://www.mtin.es/SGAS/FamiliaInfancia/infancia/ Adopcion/Adopcion.pdf.

MOHANTY, J.; NEWHILL,C.

2006 "Adjustment of international adoptees: Implications for practice and a future research agenda". Children and Youth Services Review, 28, 4: 384-395.

MORENO VILLARES, J.M.; VILLA ELÍZAGA, I.

2007 “Adopción por parejas del mismo sexo: ¿es sólo una decisión política?”. Acta Pediátrica Española, 65, 1: 1-5. 
NOY-SHARAV, Dorit

2005 "Identity Concerns in Intercountry Adoption-Immigrants as Adoptive Parents". Clinical Social Work Journal, 33, 2:173-191.

\section{OFFICIAL STATISTICS OF NORWAY}

1999 Population Statistics 1998. Documento on-line: http://www.ssb.no/emner/o2/nos befokning/nos_c607/nos_c607.pdf.

OCÓN DOMINGO, J.

2002 "Reflexiones en torno a la adopción por parejas homosexuals". Cuadernos de Trabajo Social, 15: 93-108.

OTTENBERG, Phoebe

1980 [1958] Marriage relationships in the double descent system of the Afikpo Igbo of southeastern Nigeria. Ann Arbor: University Microfilms.

PALACIOS, Jesús; BRODZINSKY, David

2010 "Adoption research: Trends, topics, outcomes". International Journal of Behavioral Development, 34, 3: 270-284.

PARKES, Peter

2003 "Fostering Fealty: A comparative Analysis of Tributary Allegiances of Adoptive Kinship”. Society for Comparative Study of Society and History, 45, 4: 741-782.

PASSIN, Herbert

1943 "The place of kinship in Tarahumara social organization". Acta Americana, 1: 360-383.

PELÁEZ, Ignacio

2001 "La adopción en el derecho comparado europeo", en A. Polaina, A. Sobrino Morrás, y A. Rodríguez Sedano (eds.), Adopción. Aspectos psicopedagógicos y marco jurídico. Barcelona: Ariel: 145-153.

PLAYFAIR, Alan

1909 The Garos. London: David Nutt.

RENAUD, M.T.

2007 "We Are Mothers Too: Childbearing Experiences of Lesbian Families". Journal of Obstetric, Gynecologic, \& Neonatal Nursing, 36, 2: 190-199.

RICKETTS, Wendell; ACHTENBERG, Roberta

1990 "Adoption and foster parenting for lesbians and gay men: creating new traditions in family", en W. Bozett y M. B. Sussman (eds.), Homosexuality and Family Relations. New York: Harrington Park Press, 83-118.

ROBY, Jini L.; MATSUMURA, Stephanie

2002 “If I Give You My Child, Aren't We Family?”. Adoption Quarterly, 5: 4, 7-31. 
SCHEPER-HUGHES, Nancy

1996 “Theft of Life: The Globalization of Organ Stealing Rumours”. Anthropology Today, 12, 3: 3-11.

SELMAN Peter

2006 "Trends in Intercountry Adoption 1998-2004: A demographic analysis of data from 20 receiving States". Journal of Population Research, 23, 2: 183-204.

2007 "Intercountry Adoption in the Twenty-first Century: an examination of the rise and fall of countries of origin”, en K. Nelson, E. Kim y M. Petersen (eds.), Proceedings of the First International Korean Adoption Studies Research Symposium. Seoul: IKAA, 55-75.

2009 "The Movement of Children for International Adoption: Developments and Trends in Receiving States and States of Origin, 1998-2004”, en L. Briggs y D. Marre (eds.), International Adoption: Global Inequalities and the Circulation of Children. New York, London: New York University Press: 32-50.

SILVERMAN, Arnold R.

1993 “Outcomes of Transracial Adoption". Future of Children, 3, 1: 104-118.

SMOLIN, David

2006 "Child laundering: how the intercountry adoption system legitimizes and incentives the practices of buying, trafficking, kidnapping, and stealing children". Wayne Law Review, 52, 1: 113-200.

STATBANK: http://www.statbank.dk.

STORM, Jacqueline M.

1990 Land of the Quinault. Taholah, Washington: Quinault Indian Nation.

TURNBULL, Colin

1965 Wayward servants: the two worlds of the African Pygmies. Garden City: The Natural History Press.

VALENTINE, Bruce; GRAY, Mel

2006 "Keeping Them Home: Aboriginal Out-of-Home Care in Australia". Families in Society, 87, 4: 537-545.

VOLKMAN, Toby Alice

2003 "Embodying Chinese Culture: Transnational Adoption in North America". Social text, 21, 1:29-55.

WEGAR, Katarina

2000 "Adoption, Family Ideology, and Social Stigma: Bias in Community Attitudes, Adoption Research, and Practice”. Family Relations, 49, 4: 363-370.

WEIL, Richard

1984 "International Adoptions: the Quiet Migration". International Migration Review, 18, 2: 276-293. 
WESTHUES, Anne; COHEN, Joyce S.

1998 "Ethnic and Racial Identity of Internationally Adopted Adolescents and Young Adults". Adoption Quarterly, 1, 4: 33-55.

YNGVESSON, Barbara

2002 "Placing the 'gift child' in Transnational Adoption". Law \& Society Review, 36, 2: 227-256. 